\title{
P-0416 - Metformin mediated gut microbiome modulation in T2D patients and controls induces specific changes in bacterial content
}

\author{
Janis Klovins ${ }^{1}$, llze Elbere ${ }^{1}$, Monta Ustinova1 ${ }^{1}$ Ivars Silamikelis ${ }^{1}$, Ilze Konrade ${ }^{2}$, Valdis Pirags \\ 1 - Latvian Biomedical Research and Study Centre \\ 2 - Riga East Clinical University Hospital
}

\section{Introduction}

Type 2 diabetes is a metabolic disease with rapidly increasing prevalence, the first choice medication for its treatment worldwide is metformin, used in clinical practice for more than 60 years. Despite its advantages, a large proportion of patients does not reach the therapeutic targets and encounter side effects. This study was conducted with aim to investigate the effects of metformin treatment on the taxonomical and functional profile of human gut microbiome and its relation to the treatment efficacy.

\section{Study design and methods}

Analyzed microbial DNA was extracted from stool samples of 35 healthy nondiabetic individuals at three time points during metformin administration: M0 - before metformin treatment, M24h 24 hours after the first metformin dose, and M7d - after a week-long metformin administration, and 50 newly-diagnosed T2D patients at two concordant time points before and week after initiation of treatment (M0, M7d) - Table 1. Healthy participants registered their diet, physical activities and side effects in special questionnaires. Information about T2D patients were collected in project specific case reports. Study design is summarized in Fig.1. Changes in the gut microbiome induced by metformin intake were assessed by metagenomic shotgun analysis employing massive parallel sequencing.

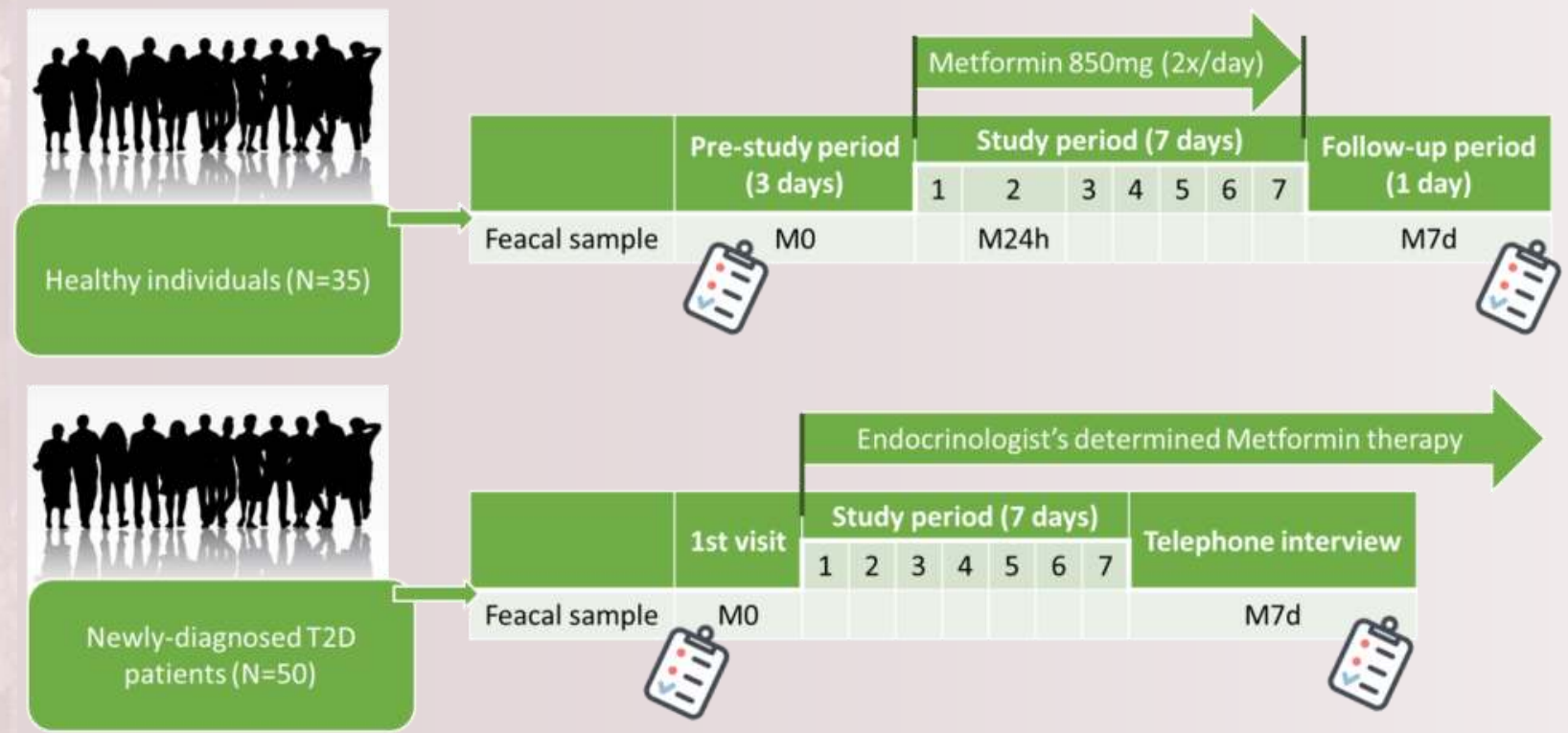

Fig.1. Design of the study.

The research has been carried out within the framework of European Regional Development Fund (ERDF), Measure 1.1.1.1 "Industry-Driven Research": „Investigation of interplay between multiple determinants influencing response to metformin: search for reliable predictors for efficacy of type 2 diabetes therapy" (1.1.1.1/16/A/091). Study has been approved by the Central Medical Ethics Committee (1/19-10-22) and State Agency of Medicines of the Republic of Latvia (17-1723), clinical trial registration number: 2016-001092-74 (www.clinicaltrialsregister.eu).

Table 1. Characterization of study subjects.

\begin{tabular}{|c|c|c|}
\hline Characteristic & $\begin{array}{l}\text { Healthy individuals, } \\
\mathrm{N}=35\end{array}$ & $\begin{array}{l}\text { T2D patients, } \\
\mathbf{N}=50\end{array}$ \\
\hline Females/ Males, n (\%) & $10(28.6 \%) / 25(71.4 \%)$ & $22(44 \%) / 28(56 \%)$ \\
\hline Age (years), average $\pm \mathrm{SD}^{1}$ & $31.5 \pm 10.2$ & $58.6 \pm 12.5$ \\
\hline $\mathrm{BMI}^{2}$, average $\pm \mathrm{SD}$ & $24.5 \pm 3.2$ & $34.8 \pm 6.7$ \\
\hline $\operatorname{ALAT}^{3}(\mathrm{U} / \mathrm{l})$, average $\pm \mathrm{SD}$ & $23.5 \pm 10.7$ & $40.3 \pm 21.8$ \\
\hline $\begin{array}{l}\text { Creatinine } \\
\text { average } \pm \mathrm{SD}\end{array}$ & $68.6 \pm 13.7$ & $68.6 \pm 13.7$ \\
\hline HbAlc (\%), average \pm SD & $5.1 \pm 0.5$ & $8.2 \pm 2.1$ \\
\hline
\end{tabular}

References:

Wu H, Esteve E, Tremaroli V, et al. (2017). Nat Med

Elbere I, Kalnina I, Silamikelis I, et al. (2018) PLoS One

Haro C, Montes-Borrego M, Rangel-Zuniga OA, et al. (2016) J Clin Endocrinol Metab 101: 233-242

Yee SW, Lin L, Merski M, et al. (2015) J Pharmacokinet Pharmacodyn 42: 463-475

\section{Results \& Discussion}

We observed significant changes in a number of bacterial taxa after a week-long administration of metformin. In both study groups, we observed a reduction in the abundance of Clostridium bartlettii, a species with controversial data on its biological role and representing a universal marker characterizing metformin use. In healthy individuals, we confirmed previously observed an increase in abundance of Escherichia coli that correlated with the highest incidence of side-effects (Fig.3), while in T2D patients increased the abundance of Parabacteroides distasonis was identified (Fig.2). This species has been recently associated with improved insulin sensitivity in obese human subjects and proven to negatively correlate with fasting blood glucose levels, therefore, possibly accounting for therapeutic effects in T2D patients.

At the functional level, we found the biosynthesis of amino acid tryptophan as well as its precursors to be among most significantly affected pathways possibly explaining previously observed association of metformin with reduced anxiety by increasing serotonin availability in the brain.

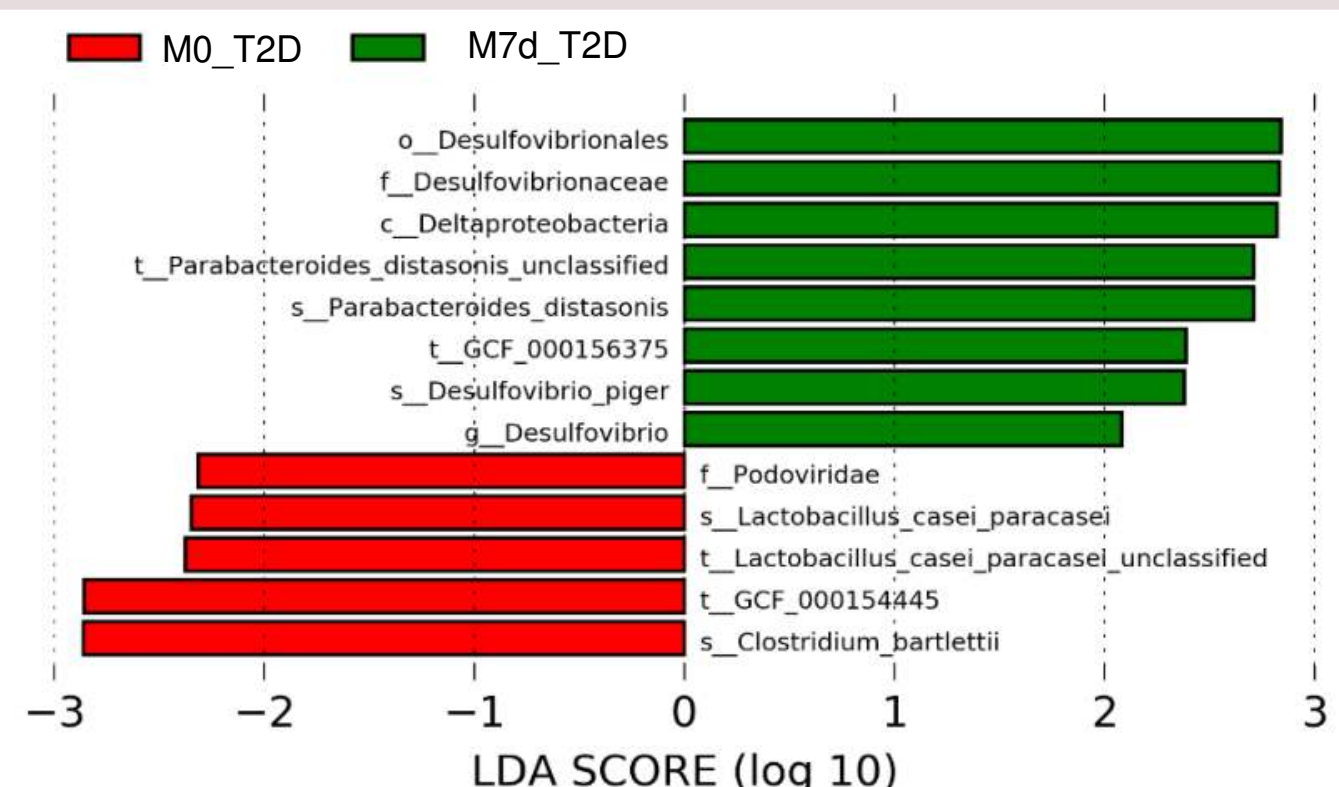

Fig.2. Differences in taxonomic composition (T2D cohort)

\section{Conclusions}

Our research brings novel information on short-term effects induced by metformin with the advantage of longitudinal data, including treatment naïve patients. In addition, the growing evidence on other therapeutic targets of metformin require more detailed information on metformin effects in non-diabetic populations. In conclusion, these results identify specific microbiome compositions that may mediate the response to metformin therapy.

The authors declare that they have no conflict of interest.

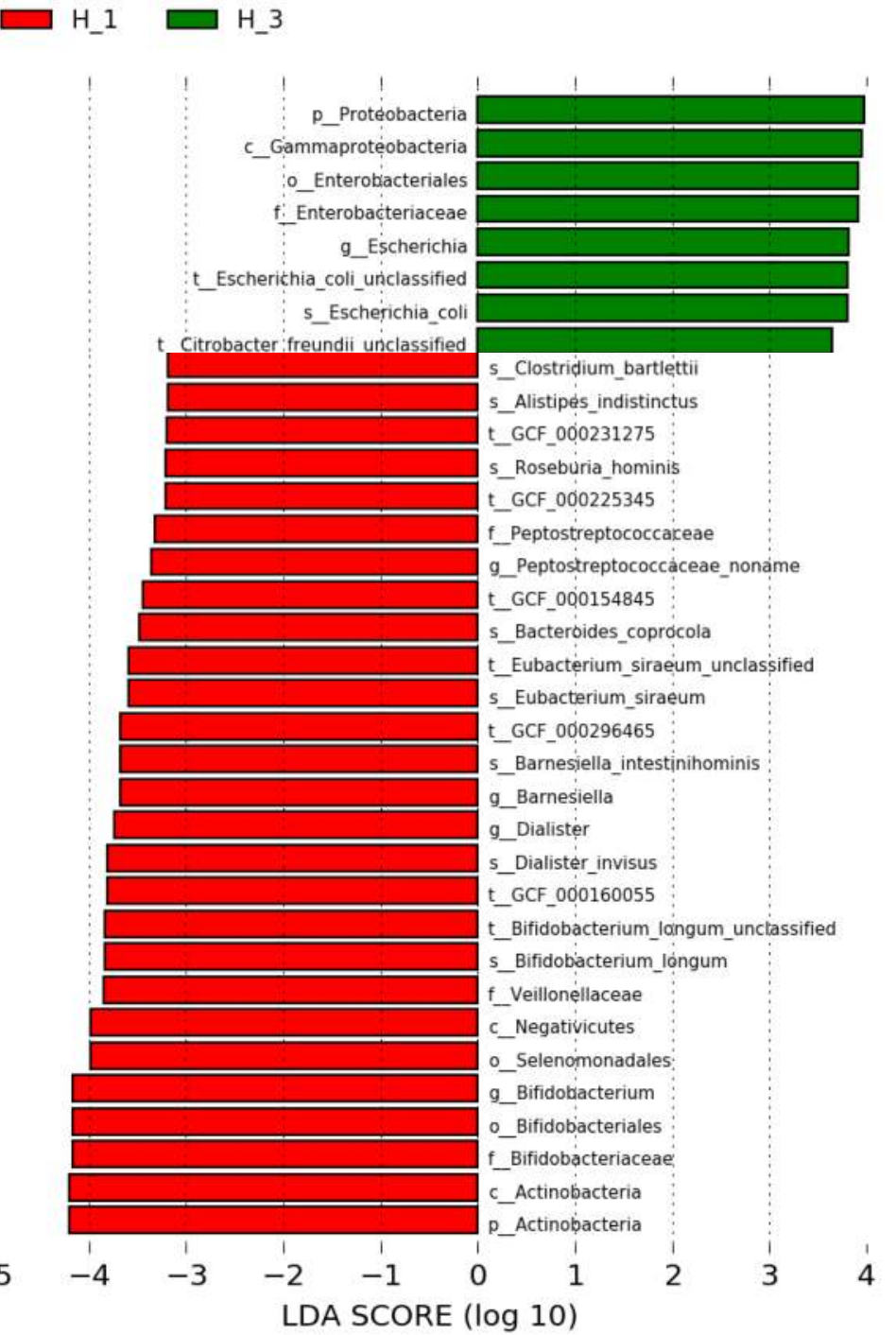

Fig.3. Differences in taxonomic composition (healthy controls) 